\title{
AOR
}

Selected Papers of \#AolR2019:

The $20^{\text {th }}$ Annual Conference of the Association of Internet Researchers

Brisbane, Australia / 2-5 October 2019

\section{MARDI GRAS THROUGH INSTAGRAM STORIES: HOW EPHEMERAL MEDIA SHAPE EVERYDAY ENGAGEMENTS WITH IDENTITY POLITICS}

\author{
Elija Cassidy \\ Queensland University of Technology \\ Ariadna Matamoros Fernandez \\ Queensland University of Technology
}

T.J. Thomson

Queensland University of Technology

A third of the world's population is active on social media and a growing number is sharing ephemeral content on such platforms. Though originally pioneered by Snapchat, Instagram has come to dominate the ephemeral media market and, as of January 2019, boasts half a billion daily Instagram Stories users (Statista, 2019). Launched roughly 2.5 years ago, in August 2016, Instagram Stories offers people a novel way of communicating through sharing photos and videos that, by default, are only available for 24 hours and then disappear. This phenomenon presents unique challenges for researchers but also demands additional attention in order to understand contemporary forms of sociability and meaning-making. This study examines people's everyday engagements with ephemeral media through an exploration of more than 400 Instagram stories created during the 2019 Sydney Gay and Lesbian Mardi Gras Festival.

While current public debates around ephemeral content revolve around how bad users have turned to the likes of Instagram and Snapchat in order to circumvent platform moderation (Gerrard, 2018) or boost engagement with brands (Trounce, 2018), we are interested in broader dynamics involved in the shift to temporary media for everyday social media communication. What factors drive user interaction with ephemeral 
content? How does the immediacy or 'liveness' (Amaral, 2018) of temporary media reconfigure the workings of digital publics, identity politics, and individual user experiences?

This paper presents initial findings from phase one of an exploratory project that seeks to address these questions through the lens of ephemeral media on Instagram, and, in particular, through the practices associated with use of Instagram Stories around a key public event. We use the Sydney Gay and Lesbian Mardi Gras for its concrete/timebound nature and because it brings together history, politics, corporate, state, and community actors. This event is also replicated in one fashion or the other around the world; thereby opening up opportunities for future comparative studies. The event allows us to examine the nature of community engagement with ephemeral media, and the potential challenges and opportunities for identity politics brought about through Instagram Stories.

The latest cutting-edge research in the digital media field places Instagram at the centre of emerging social media research (Serafinelli, 2018; Leaver, Highfield, \& Abidin, 2019). However, research specifically on Stories, as a common feature of this platform, is scarce. Hence, this paper contributes to further extending developments in research on ephemeral media by examining user practices with Instagram Stories and their implications for everyday communication from a critical cultural studies perspective. It is guided by the following research questions:

RQ1: How are ephemeral media changing user practices on Instagram?

RQ2: What challenges and opportunities exist around platform affordances, like Stories, for Instagram users (e.g. modes of expression, loss of cultural archive, spread of false information, community engagement issues); and RQ3: How does the above play out in the context of an event focussed around identity politics, such as the Sydney Gay and Lesbian Mardi Gras?

\section{Method}

Our overarching project takes an ethnographic approach to the study of digital media, integrating content analysis of Instagram stories with semi-structured interviews with relevant public actors involved in Mardi Gras who used Instagram Stories as part of their engagement with the event.

This paper emerges from the first phase of the data gathering, involving the collection and analysis of more than 400 Instagram Stories published on the platform during Mardi Gras. To identify a manageable sample of Stories to examine, the research team first started with all 942 accounts the official @sydneymardigras Instagram account followed in January 2019. All private accounts were removed from the sample along with accounts not actively posting Stories content. This was determined by examining accounts twice within the span of one week, with 24 hours in between examinations, to 
see if users had posted a story in the previous 24 hours. Only accounts which had posted Stories during both checks were classified as 'active' posters of ephemeral Instagram content. This resulted in 135 accounts. Based on the remaining list of 135 accounts the team deductively created the following five category types:

1) official organising committee and members of @sydneymardigras

2) official partners/sponsors

3) public figures

4) organisations

5) members of the general public

A random sample from each account type was created to generate a sample of 30 accounts. Every day of the official Sydney Gay and Lesbian Mardi Gras Festival (Feb. 15-March 3, 2019) all Stories posted to these accounts were saved by the research team using built-in smartphone screen recording technology. The resulting body of material was analysed in order to better understand how users are employing the Stories feature in their everyday communication and to engage in identity politics. We identified thematic patterns in content posted, hashtags used, and actors tagged. In phase two of the project the team will interview users of the above accounts to more fully understand their experiences using and engaging with Instagram Stories. Users' reflexive participation in these interview sessions will construct a feedback loop, to work as a check against the veracity of the observations made in phase one of the project.

\section{Contribution}

In this project we aim to examine the place of ephemeral media in everyday communication. Some exploratory work using interviews has been done in this vein but few studies have attempted an analysis of users' practices through Instagram Stories themselves.

Preliminary findings show that people engaged with Stories for different purposes during the Mardi Gras celebration. Users created ephemeral content showing nudity; promoting products from advertisers; political talk; and as means to broadcast what was happening on TV with overlaid stickers and comments. Users also employed Stories to connect with their audiences through personal "confessions", or direct-to- camera narrations. In these cases, Instagram Stories' unique "ask me a question" feature further afforded direct communication with audiences, since users would generate more stories with their answers to these questions. Common internet practices like self-deprecating humour (to give a sense of authenticity) were also salient in user practices with ephemeral media.

Through this empirical work, we aim to start a public conversation around the Implications of "losing" ephemeral media and how this might impact archival practices for the study of historical events that get heavily mediated through digital media. The 
larger project from which this paper emerges also contributes more broadly to digital media studies by addressing how to effectively study ephemeral digital objects, like Instagram Stories.

\section{References}

Amaral, L. (2018). Liveness beyond live video: immediacy and presence on Instagram Stories. Paper presented at Instagram Conference 2018: Studying Instagram Beyond Selfies. Middlesex University, London, 1 June, 2018.

Gerrard, Y. (2018). Beyond the hashtag: Circumventing content moderation on social media. New Media \& Society, 20(12), 4492-4511.

Leaver, T., Highfield, T., \& Abidin, C. (2019). Instagram: Visual Social Media Cultures. Cambridge: Polity Press.

Serafinelli, E. (2018). Digital Life on Instagram: New Social Communication of Photography. Bingley, West Yorkshire: Emerald Publishing Limited.

Statista. (2019). Number of daily active Instagram Stories users from October 2016 to January 2019 (in millions). Retrieved March 1, 2019, from https://www.statista.com/statistics/730315/instagram-stories-dau/

Trounce, D. (2018). Ephemeral Content: Everything Marketers Need to Know. Retrieved March 1, 2019, from Search Engine Journal, https://www.searchenginejournal.com/ephemeral-content/238589/\#close 